\title{
Schwere Akne unter Therapie eines metastasierten kolorektalen Karzinoms mit monoklonalem Anti-EGF-Rezeptor-Antikörper Cetuximab (Erbitux ${ }^{\circledR}$ )

\author{
L. Kowalzick ${ }^{1}$ \\ C. Lohse ${ }^{2}$ \\ H. Ziegler ${ }^{1}$ \\ F. W. Busch 2
}

\author{
Severe Acne Following Treatment of Metastatic Colorectal Carcinoma with \\ Monoclonal Anti-EGF-Receptor Antibody Cetuximab
}

\section{Zusammenfassung}

Seit kurzem werden Inhibitoren des Rezeptors für epidermalen Wachstumsfaktor (EGF) in der Therapie fortgeschrittener, chemotherapieresistenter Karzinomerkrankungen, wie dem kolorektalen Karzinom und dem nicht-kleinzelligen Bronchialkarzinom eingesetzt. Aus den Zulassungsstudien ist bekannt, dass bei der Mehrzahl der Patienten hierunter Nebenwirkungen am Hautorgan auftreten. Die häufigste kutane Nebenwirkung bei 29 bis 39\% der Patienten ist das Auftreten akneiformer Hauterscheinungen. Wir berichten über einen 64-jährigen Patienten mit metastasiertem kolorektalen Karzinom, das auf die StandardSecond-line-Chemotherapie mit Irinotecan nicht mehr ansprach und daher zusätzlich mit Cetuximab $\left(\right.$ Erbitux $\left.^{\circledR}\right)$, einem monoklonalen Antikörper gegen den EGF-Rezeptor behandelt wurde. Während des 2. Therapiezyklus (wöchentliche Applikation) entwickelte er binnen eines Tages massive hochentzündliche Hautveränderungen im Gesicht, den Schultern und in der vorderen und hinteren Schweißrinne, die morphologisch das Bild einer Acne fulminans erreichten. Noch ohne spezifische Therapie besserte sich das Krankheitsbild spontan bereits nach wenigen Tagen und klang unter symptomatischer Therapie binnen 2 Wochen weitgehend ab. Die enge zeitliche Korrelation der Gabe von EGF-Rezeptor-Antagonisten und dem Auftreten von Akne könnte für eine Rolle des EGF/EGF-Rezeptor-Systems bei der Pathogenese dieser Erkrankung sprechen.

\section{Abstract}

Recently, inhibitors of epidermal growth factor (EGF) receptor were approved by health authorities for treatment of metastastatic carcinomas including colorectal and non-small cell lung carcinoma not responding to standard chemotherapies. It is known from clinical studies that most of the treated patients developed unwanted drug induced side effects of the skin. The cutaneous side effect occurring most frequently is the eruption of acne lesions in 29 to $39 \%$ of the patients treated. We report on a 64 years old male patient with metastatic colorectal carcinoma not responding anymore to standard second line chemotherapy with irinotecan and then additionally was treated with cetuximab $\left(\right.$ Erbitux $\left.^{\circledR}\right)$, a monoclonal antibody directed against the EGF receptor. During the second therapy cycle (weekly administrations) he developed within a day severe inflammatory skin lesions of the face, the shoulders, the back and his breast resembling morphologically acne fulminans. Without specific therapy the skin disease improved spontaneously within several days and cleared under symptomatic therapy within 2 weeks. This side effect could lead to the assumption of a role of the EGF/EGF receptor system in the pathogenesis of acne.

Institutsangaben

${ }^{1}$ Klinik für Hautkrankheiten und Allergologie, HUMAINE Vogtland-Klinikum Plauen, (Chefarzt: Prof. Dr. med. habil. L. Kowalzick)

${ }^{2}$ Medizinische Klinik, Klinikum Hof (Leitende Ärzte: Prof. Dr. med. F. W. Busch, Dr. med. H. Militzer, Prof. Dr. med. Th. Podszus)

Korrespondenzadiresse

Prof. Dr. med. habil. Lutz Kowalzick · Klinik für Hautkrankheiten und Allergologie · HUMAINE VogtlandKlinikum Plauen·Postfach 100153 · 08505 Plauen·E-mail: lutz.kowalzick@web.de

Bibliografie

Akt Dermatol 2004; 30: 474-477 @ Georg Thieme Verlag KG Stuttgart · New York .

DOI 10.1055/s-2004-825877 · ISSN 0340-2541 
Vor kurzem wurde in der Schweiz und in den USA ein chimärer monoklonaler $\operatorname{IgG}_{1}$-Antikörper gegen EGF-Rezeptor, Cetuximab $\left(\right.$ Erbitux $^{\circledR}$ ) zur Therapie des metastasierten kolorektalen Karzinoms zugelassen. Er wird eingesetzt, wenn der Patient auf das Standard-Second-line-Chemotherapeutikum, die Platinverbindungen Carboplatin oder Irinotecan (Campto $\left.{ }^{\circledR}\right)$ nicht, bzw. nicht mehr anspricht und die Tumorzellen EGF-Rezeptor exprimieren. In klinischen Studien an mehreren hundert Patienten sprachen auf eine Kombination von Cetuximab und Irinotecan zwischen 21 und 50\% der Patienten an. Eine Monotherapie mit dem Antikörper führte zu einer Ansprechrate von nur 5 bis $12 \%$. Die mittlere Zeit zur Progression fand sich in der Kombinationstherapie gegenüber der Monotherapie signifikant verlängert, die mittlere Überlebenszeit ebenfalls, wenngleich nicht signifikant, um ca. zwei Monate verlängert $[4,14]$. Auch in Deutschland wird das Präparat bereits im Rahmen individueller Heilversuche klinisch eingesetzt.

In den Studien, die zur Zulassung führten, wurden sehr häufig (in über $80 \%$ ) kutane Nebenwirkungen beobachtet. Die häufigsten Nebenwirkungen an der Haut sind Akne mit ca. 29\%, gefolgt von Hauttrockenheit (Xerosis, Desquamatio) mit ca. 26\%, Nagelerkrankungen (Paronychie, Unguis incarnatus) mit ca. $12 \%$ und Arzneimittelexantheme bei $15 \%$ unter Kombinationstherapie mit Irinotecan und bei $8 \%$ unter Monotherapie. Haarausfall trat praktisch nur in der Kombination mit dem Zytostatikum bei $24 \%$ der Patienten auf $[4,11]$.

Wir berichten hier über Klinik und Verlauf schwerer Acne-fulminans-artiger Hautveränderungen bei einem 64-jährigen Patienten mit metastasiertem kolorektalen Karzinom, der mit einer Kombinationstherapie mit Irinotecan und Cetuximab behandelt wurde.

\section{Kasuistik}

Ein 64-jähriger Patient mit metastasiertem kolorektalen Karzinom erhielt erstmalig eine Infusion mit der Initialdosis von $400 \mathrm{mg} / \mathrm{m}^{2}$ Körperoberfläche des monoklonalen Anti-EGF-Rezeptor Antikörper Cetuximab. Als empfohlene Begleitmedikation erhielt der Patient einmalig $2 \mathrm{mg}$ des Antihistaminikums Clemastin (Tavegil ${ }^{\circledR}$ ) sowie $100 \mathrm{mg}$ Prednisolon i.v. zur Prophylaxe einer anaphylaktischen Reaktion. Weitere Glukokortikosteroide erhielt der Patient im Zusammenhang mit dieser Chemoimmuntherapie nicht. Der Patient gab an, in seiner Pubertät, Adoleszenz, oder auch später nicht an nennenswerten Symptomen einer Acne vulgaris gelitten zu haben. Als er eine Woche später zur zweiten vorgesehenen Infusion mit der Erhaltungsdosis von $250 \mathrm{mg} / \mathrm{m}^{2}$ Körperoberfläche erschien, war es bereits prästernal zur Entwicklung einzelner follikulärer Papeln und Pusteln gekommen. Bereits einen Tag später kam es zu einer massiven Aussaat papulöser und pustulöser Hautveränderungen im Gesicht, an den Schultern und an Brust und Rücken. Im Gesicht kam es zu starkem Nässen der Hautveränderungen. Allgemeinsymptome wie Fieber oder Polyarthritis seien nicht aufgetreten. Ohne spezifische symptomatische Therapie sei es im Verlauf einer Woche bereits zu einer spontanen Besserung der Haut-

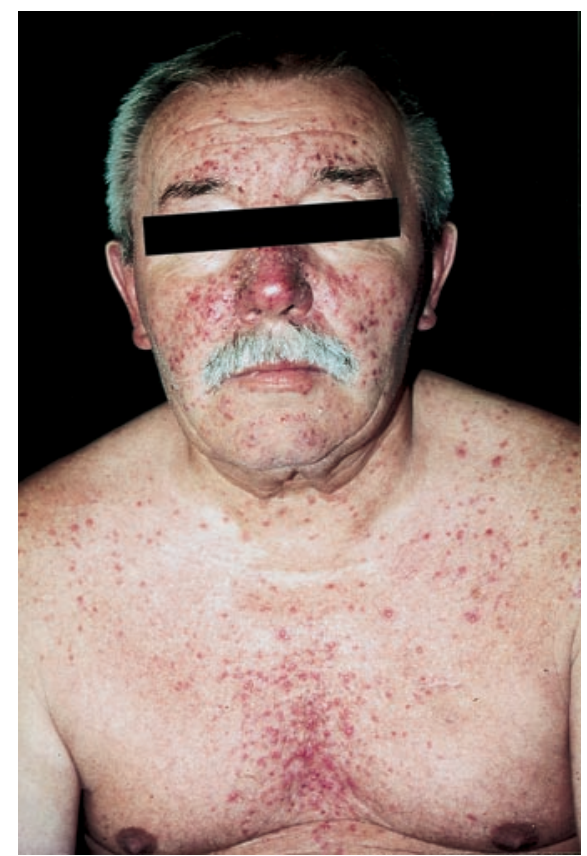

Abb. 1 64-jähriger Patient mit metastasierendem kolorektalen Karzinom 10 Tage nach der zweiten Infusion von Cetuximab, einem monoklonalen Antikörper gegen den Rezeptor für epidermalen Wachstumsfaktor (EGF). Eruption akneiformer Hautveränderungen mit Komedonen, follikulär gebundenen $\mathrm{Pa}$ peln und Pusteln in Acne-vulgaris-typischen Lokalisationen im Gesicht, an den Schultern und der vorderen Schweißrinne.

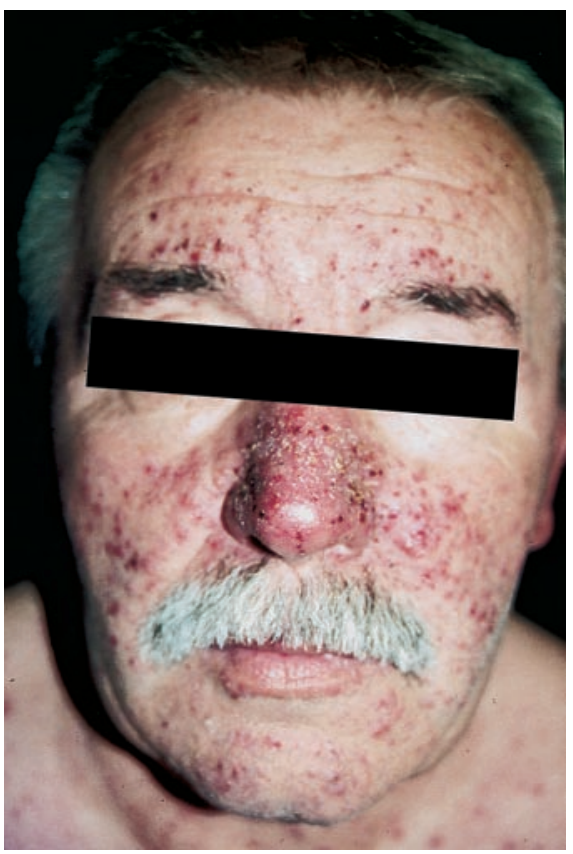

Abb. 2 Detailaufnahme des Gesichts zum gleichen Zeitpunkt. Deutliche Ausbildung von hämorrhagischen und serösen Krusten sowie Schuppung besonders im zentrofazialen Bereich. Nach Angabe des Patienten waren die Hautveränderungen dort ab dem ersten Tag nach der zweiten Infusion von Cetuximab stark nässend und jetzt ohne spezifische Therapie bereits deutlich rückläufig.

erscheinungen gekommen. Gleichwohl waren die Hautsymptome noch so ausgeprägt, dass die vorgesehene zweite Erhaltungsdosis Cetuximab, auch auf Wunsch des Patienten selbst, zunächst nicht gegeben wurde, und eine dermatologische Vorstellung erfolgte. Zehn Tage nach der letzten Cetuximab-Infusion fanden sich an der Stirn, der Nase, den Wangen, dem Kinn, den Schultern sowie der vorderen und hinteren Schweißrinne disseminierte Komedonen sowie follikulär gebundene Papeln und Pusteln, die teilweise großflächig konfluierten. Insbesondere im Bereich der Nase und Wangen fanden sich weißlich-gelbliche Krusten (Abb.1-3). Auffällig war daneben eine konjunktivale Rötung wie bei Rosazea. Es wurde eine systemische Therapie mit Minocyclin $2 \times 50 \mathrm{mg} /$ die sowie eine Lokaltherapie mit Erythromycin $4 \%$ igem Hydrogel $2 \times$ tgl. eingeleitet und empfohlen, hierunter die Therapie mit Cetuximab ggf. nach empfohlener Do- 


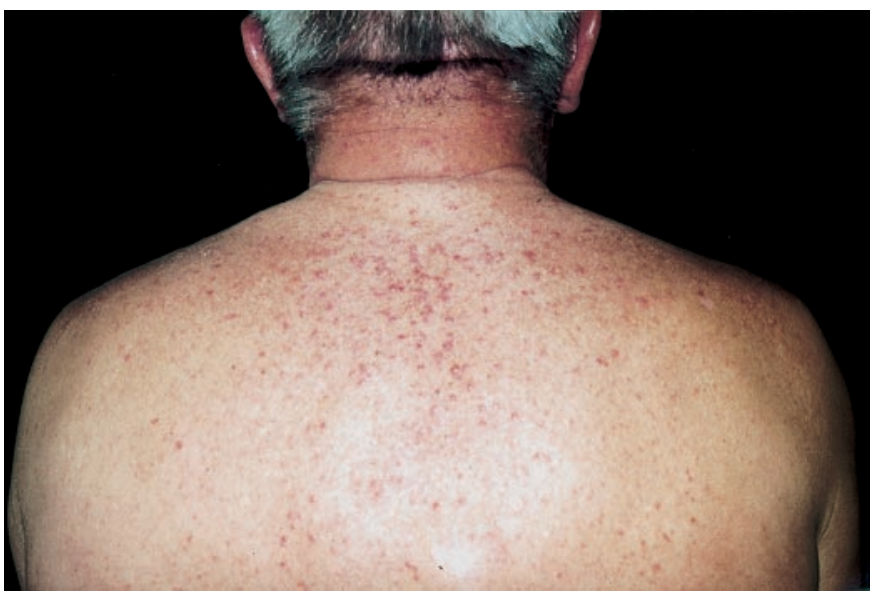

Abb. 3 Ähnlicher Befund wie an der vorderen hier auch an der hinteren Schweißrinne und, Akne-vulgaris-untypisch, zusätzlich im Nackenbereich und am Hinterkopf.

sisanpassung fortzusetzen, solange keine Allgemeinsymptomatik mit Fieber oder Polyarthritis während der Akneschübe aufträte. Unter der genannten symptomatischen Therapie kam es binnen nur einer Woche zu einer weitgehenden Rückbildung der akneiformen Hautveränderungen, so dass die Fortsetzung der Behandlung mit Cetuximab möglich erschien.

\section{Diskussion}

Bei manchen Tumorentitäten ist die EGF-Rezeptor-Signalübertragung beteiligt an der Erhöhung der Überlebensfähigkeit, der Zellzyklusprogression, der Zellmigrations- und Invasionsfähigkeit von Tumorzellen, sowie deren Fähigkeit, Angiogenesefaktoren zu produzieren. Die Expression von EGF-Rezeptor bei diesen Erkrankungen korreliert mit einer erhöhten Bildung von Metastasen und einer verminderten Überlebenszeit [1].

Seit kurzem werden Inhibitoren des Rezeptors für EGF in der Therapie fortgeschrittener, chemotherapieresistenter Karzinomerkrankungen, wie dem kolorektalen Karzinom und dem nichtkleinzelligen Bronchialkarzinom eingesetzt. Zum einen handelt es sich um einen monoklonalen Antikörper gegen die extrazelluläre Domäne des Rezeptors (Cetuximab) [14], zum anderen um Gefitinib (Iressa ${ }^{\circledR}$ ), einen oral einzunehmenden spezifischen Hemmer von Tyrosin-spezifischen Proteinkinasen und somit der EGF-Rezeptor Signal-Transduktion [7]. Letzteres Präparat ist durch die FDA 2003 zur Therapie lokal fortgeschrittener oder metastasierter nicht-kleinzelliger Bronchialkarzinome, die nicht bzw. nicht mehr auf eine Chemotherapie mit Platin- oder TaxanPräparaten ansprechen, zugelassen worden [2].

Diese Präparate blockieren die o.g. tumorfördernden Effekte der EGF-Rezeptor Signal-Transduktion [1]. Der monoklonale Antikörper führt darüber hinaus zu einer Internalisierung und Down-Regulation des Rezeptors sowie zu einer Vermehrung der antikörperabhängigen Zytotoxizität natürlicher Killerzellen [4].

Interessanterweise ist, trotz ihres ganz unterschiedlichen Angriffspunktes am EGF-Rezeptor, aus den Studien mit beiden Präparaten bekannt, dass bei den Patienten hierunter dosisabhängig häufig Akne als kutane Nebenwirkung auftritt. Die Angaben über die Häufigkeit schwanken von $17 \%$ bis $86 \%$ (im Mittel 29\%) $[4,9,14]$ für Cetuximab und werden mit $39 \%$ für Gefitinib angegeben [8]. Diese Hautveränderungen beginnen typischerweise im Gesicht und am oberen Rumpf jedoch auch am Kapilitium. In bis $\mathrm{zu} 1 / 5_{5}$ der Fälle würden die akneiformen Hauterscheinungen zumindest $50 \%$ der Körperoberfläche betreffen. Nach Absetzen der Präparate bilden diese sich ohne Narbenbildung zurück. Möglicherweise korreliert diese Nebenwirkung mit dem therapeutischen Ansprechen der Tumorerkrankung [14].

Mit Ausnahme der fehlenden Allgemeinsymptomatik mit Fieber oder Polyarthritis entsprach der klinische Befund im vorliegenden Fall mit „blitzartigem“ Beginn und starker großflächiger exsudativer Komponente ohne Fuchsbau-Komedonen und Zysten und eher wenig Komedonen einer Acne fulminans [13].

Bemerkenswert ist der rasche Beginn der Aknesymptomatik binnen einer Woche nach Therapiebeginn und die rasche spontane Rückbildung nach Absetzen bzw. Aussetzen der Therapie. Ein Befund einer nicht-medikamentös induzierten schweren und stark exsudativen Akne, der der Schwere nach mit dem vorliegenden unter Cetuximab aufgetretenen Fall vergleichbar wäre, würde unter einer symptomatischen Therapie allein mit Minocyclin intern und Erythromycin extern nicht binnen 2 Wochen abheilen. Die Serumhalbwertszeit von Cetuximab nach Infusion beträgt 70 bis 100 Stunden [4]. Diese Umstände sprechen für einen möglichen unmittelbaren Einfluss des EGF/EGF-Rezeptorsystems auf die Pathogenese der Akne.

Der aknefördernde Effekt einer Blockierung der EGF-Rezeptor Signaltransduktion ist auf den ersten Blick überraschend. So führte an Talgdrüsen in vitro die Addition von EGF zu einer Desorganisation der Keratinozyten und zu einer Ruptur des Infundibulums wie bei schwererer, purulenter Akne [5]. Auf der anderen Seite konnte jedoch an Talgdrüsen in vitro gezeigt werden, dass EGF die Talgproduktion hemmt und eine De-Differenzierung von Sebozyten in einen Keratinozyten-ähnlichen Phänotyp induziert und eine Rolle bei der klinischen Spontanremission von Akneläsionen spielt [3]. In vitro führt EGF an isolierten Haarfollikeln zu einem Übergang von der Anagen- zur Katagenphase des Haarzyklus [12]. In der Folge kommt es zu einer Expression von MHC Klasse-I-Antigenen im unteren Follikel, zur Makrophageninfiltration und verminderten Suppression von reaktiven Sauerstoffspezies und somit zur Follikeldestruktion [6,10,12]. In der Tat können EGF-Rezeptor-Antagonisten in vivo zu einer exzessiven follikulären Hyperkeratose mit Obstruktion der Follikelostien und darüber hinaus zu einer Beschleunigung des Haarzyklus führen [15]. Diese Mechanismen könnten das Auftreten von schweren Akneschüben als Nebenwirkung der Therapie mit EGFRezeptor-Antagonisten erklären.

\section{Literatur}

${ }^{1}$ Baselga J. Why the epidermal growth factor receptor? The rationale for cancer therapy. Oncologist 2002; 7, Suppl 4: 2-8

${ }^{2}$ Cohen MH, Williams GA, Sridhara R, Chen G, McGuinn WD, Morse D, Abraham S, Rahman A, Liang C, Lostritto R, Baird A, Pazdur R. United States food and drug administration approval of gefinitimib (ZD1839; Iressa) tablets. Clin Cancer Res 2004; 10: 1212 - 1218 
${ }^{3}$ Downie MM, Sanders DA, Kealey T. Modelling the remission of individual acne lesions in vitro. Br J Dermatol 2002; 147: 869-878

${ }^{4}$ Fachinformation des Arzneimittelkompendiums der Schweiz. Basel: Documed, 2003: 1-15

${ }^{5}$ Guy R, Green MR, Kealey T. Modelling acne in vitro. J Invest Dermatol 1996; 106: $176-182$

${ }^{6}$ Hansen LA, Alexander N, Hogan ME, Sundberg JP, Dlugosz A, Threadgill DW, Magnuson T, Yuspa SH. Genetically null mice reveal a central role for epidermal growth factor receptor in the differentiation of the hair follicle and normal hair development. Am J Pathol 1997; 150: $1959-1975$

${ }^{7}$ Kris MG, Natale RB, Herbst RS, Lynch TJ, Prager D, Belani CP, Schiller JH, Kelly K, Spirindonidis J, Sandler A, Albain KS, Cella D, Wolf MK, Averbuch SD, Ochs JJ, Kay AC. Efficacy of gefitinib, an inhibitor of the epidermal growth factor receptor tyrosine kinase, in symptomatic patients wit non-small lung cancer: a randomized trial. JAMA 2003; 290: $2149-2158$

${ }^{8}$ Lee MW, Seo CW, Kim SW, LeeHW, Choi JH, Moon KC, Koh JK. Cutaneous side effects in non-small cell lung cancer patients treated with Iressa (ZD1839), an inhibitor of epidermal growth factor. Acta Demato Venereol 2004; 84: 22 - 26

${ }^{9}$ Motzer RJ, Amato R, Todd M, Hwu WJ, Cohen R, Baselga J, Muss H, Cooper M, Yu R, Ginsberg MS, Needle M. Phase II trial of antiepidermal growth factor receptor antibody C225 in patients with advanced renal carcinoma. Invest New Drug 2003; 21: 99-101

${ }^{10}$ Nanney LB, Magid M, Stoscheck CM, King LE. Comparison of epidermal growth factor binding and receptor distribution in normal human epidermis and epidermal appendages. J Invest Dermatol 1984; 83: 385 393

${ }^{11}$ Needle MN. Safety experience with IMC-C225, an anti-epidermal gowth factor receptor antibody. Semin Clin Oncol 2002; 29,S14: 55 60

12 Philpott MP, Kealy T. Effects of EGF on the morphology and patterns of DNA synthesis in isolated human hair follicles. J Invest Dermatol 1994; 102: 186 - 191

${ }^{13}$ Plewig G, Kligman AM. Acne and Rosacea. $3^{\text {rd }}$ Edition. Berlin: SpringerVerlag, 2000: $342-351$

${ }^{14}$ Saltz LB, Meropol NJ, Loehrer PJ, Needle MN, Kopit J, Mayer RJ. Phase II trial of antiepidermal growth factor receptor antibody C225 in patients with refractory colorectal cancer that express the epidermal growth factor receptor. J Clin Oncol 2004; 22: 1201 -1208

15 VanDoorn R, Kirtschig G, Scheffer E, Stoof TJ, Giaccone G. Follicular and epidermal alterations in patients treated with ZD1839 (Iressa), an inhibitor of the epidermal growth factor receptor. Br J Dermatol 2002; 147: $598-601$

\section{Buchbesprechung}

\section{Ästhetische Dermatologie; Dermatologie und Kosmetik der Menschen mit schwarzer Haut und der Mischlinge \\ Eric-David Aumjaud}

Médecine esthétique, dermatologie et cosmétologie des sujets noirs et métissés. Cachan, France: E. M. Inter Lavoisier. 75,- $€$. ISBN: 274300648x

Ein gewichtiges Problem wird hier angesprochen und von einem Spezialisten aus Paris in hervorragender Weise dargestellt, ausführlich und doch knapp, verständlich und nicht vereinfachend, kompetent und nicht schulmeisterlich, und vor allem sachdienlich und vorsichtig. Es strahlt das Buch die Anteilnahme an den Patienten, die Rücksicht auf deren Anliegen und die Vorsicht und Zurückhaltung vor gewagten Vorschlägen geradezu aus. Es werden die anatomischen und funktionellen Unterschiede zur weißen Haut dargestellt und belegt, die Besonderheiten der schwarzen Haut aufgeführt und die vordringlichen Probleme angesprochen: Pigmentunterschiede genuin und hervorgehoben bei Aussetzen der Lichtstimulierung im Gesicht, an den Oberschenkeln und natürlich palmo-plantar, sowie die Xerose der trockenen Haut und die Haarprobleme.

Akne, Follikulitis, Rollhaarzysten, Keloide und Narben sind mit sanften und effektiven Therapien in den Vordergrund gerückt, während erwartungsbeladene, jedoch nicht sichere Methoden und Indikationen als solche gekennzeichnet oder gar abgeraten werden. Die Bilder sind reichlich, informativ und technisch ansprechend, rechtfertigen aber den erstaunlichen Preis nicht ausreichend.

Es handelt sich bei der hohen Migration in Europa um einen sehr wichtigen Leitfaden, der für Frankreich besonders bedeutungsvoll ist, betrifft er dort doch über $10 \%$ der Menschen.

E. G. Jung, Heidelberg 